\title{
Deutschsprachiger AOTrauma-Master-Kurs in Davos
}

Florian Gebhard

\section{Vom Sonntag, 03.12., bis Donnerstag, 07.12.2017 findet in Davos der traditionelle deutschsprachige AOTrauma-Master-Kurs statt, gemeinsam organisiert von der AOTrauma Deutschland, Österreich und Schweiz. Die Leitung haben Pol Rommens (Mainz), Michael Plecko (Graz) und Andreas Platz (Zürich). Inhaltlicher Schwerpunkt sind komplexe Fraktursituationen und deren Begleitverletzungen.}

In der Vergangenheit hat sich deutlich gezeigt, dass die Diskussion komplexer Fälle intensiver geführt wird, wenn diese auf Deutsch stattfindet. Geschätzt wurde auch der intensive Austausch mit den Kollegen und Kolleginnen aus Österreich und der Schweiz. Abgesehen vom internationalen Flair während der Davoser Trauma-Kurswoche mit über 1000 Teilnehmenden und Faculty aus aller Welt sind die Self-directed Learning Modules nur in Davos während der AOTrauma-Kurswoche möglich.

Die Teilnehmenden sollten folgende Kriterien erfüllen:

- Teilnahme am AOTrauma-Prinzipien-Kurs und dem Fortgeschrittenenkurs für operative Frakturbehandlung

- Aktuelle Tätigkeit in der Traumaversorgung in einer unfallchirurgischen/orthopädischen Abteilung

- mindestens 8-10 Ausbildungsjahre (Facharzt für Chirurgie, Orthopädie oder Unfallchirurgie und Orthopädie)

Aufgrund einer fundamentalen Neuausrichtung der Davos AO-Kurse könnte dies die letzte Chance sein, einen deutschsprachigen AOTrauma-Master-Kurs in Davos besuchen zu können.

Die Anmeldung ist möglich unter:

www.aodavoscourses.org

Florian Gebhard, Ulm

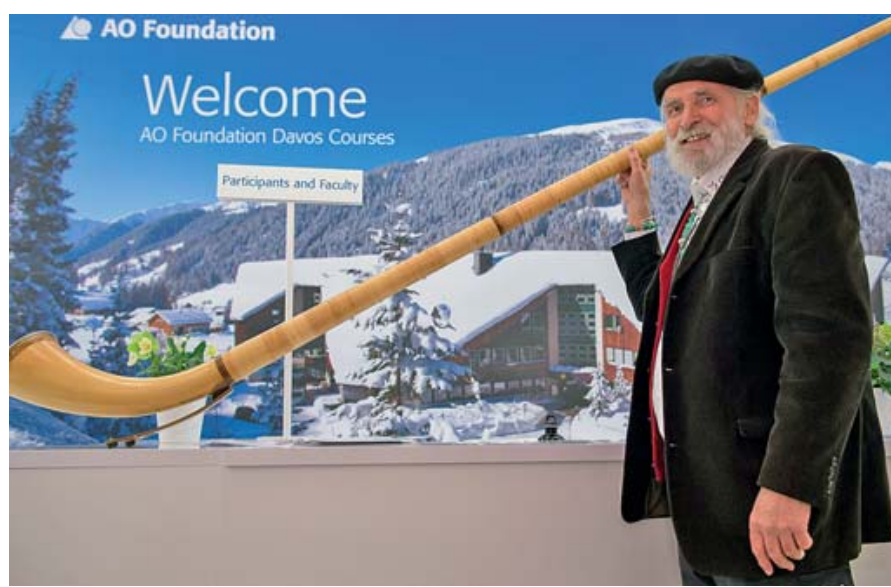

- Abb. 1 Willkommen in der AO-Welt in Davos. Bild: AO Foundation.

Bibliografie

DOI https://doi.org/10.1055/s-0043-116400 OP-JOURNAL 2017; 33: 198 @ Georg Thieme Verlag KG Stuttgart · New York ISSN 0178-1715 